OPEN ACCESS

Edited by:

Patrícia Mendonça Rijo, Universidade Lusófona Research Center for Biosciences \& Health

Technologies, Portugal

Reviewed by:

Ana Fernandes,

Universidade Lusófona Research

Center for Biosciences \& Health

Technologies, Portugal

Qiyang Shou,

Zhejiang Chinese Medical University,

China

Qinhong Xu,

First Affiliated Hospital of Xi'an Jiaotong University, China

*Correspondence:

Yang Liu

Liu-yang@xjtu.edu.cn

Gang Cao

chenxijiaoda@163.com

Specialty section:

This article was submitted to Pharmacology of Anti-Cancer Drugs,

a section of the journal

Frontiers in Oncology

Received: 21 August 2020

Accepted: 21 October 2020

Published: 02 December 2020

Citation:

Chen S, Shen J, Zhao J, Wang J, Shan T, Li J, Xu M, Chen X, Liu Y and Cao G (2020) Magnolol Suppresses

Pancreatic Cancer Development In Vivo and In Vitro via Negatively Regulating TGF- $\beta /$ Smad Signaling.

Front. Oncol. 10:597672.

doi: 10.3389/fonc.2020.597672

\title{
Magnolol Suppresses Pancreatic Cancer Development In Vivo and In Vitro via Negatively Regulating TGF- $\beta /$ Smad Signaling
} Shuo Chen ${ }^{1}$, Jiaqi Shen ${ }^{2}$, Jing Zhao ${ }^{3}$, Jiazhong Wang ${ }^{1}$, Tao Shan ${ }^{1}$, Junhui $L i^{1}$, Meng $X u^{1}$,
Xi Chen ${ }^{1}$, Yang Liu ${ }^{1 *}$ and Gang Cao ${ }^{1 *}$

${ }^{1}$ Department of General Surgery, The Second Affiliated Hospital of Xi'an Jiaotong University, Xi'an Jiaotong University, Xi'an, China, ${ }^{2}$ School of Life Science, Xiamen University, Xiamen, China, ${ }^{3}$ School of Science, Xi'an Jiaotong University, Xi'an, China

Magnolol, a hydroxylated biphenyl extracted from Magnolia officinalis, has recently drawn attention due to its anticancer potential. The present study was aimed to explore the effects of Magnolol on restraining the proliferation, migration and invasion of pancreatic cancer in vivo and in vitro. Magnolol showed significant anti-growth effect in an orthotopic xenograft nude mouse model, and immunohistochemical staining of the xenografts revealed that Magnolol suppressed vimentin expression and facilitated E-cadherin expression. The cytoactive detection using CCK-8 assay showed Magnolol inhibited PANC-1 and AsPC-1 concentration-dependently. Scratch healing assay and the Transwell invasion assay proved the inhibiting effects of Magnolol on cellular migration and invasion at a non-cytotoxic concentration. Western blot and rt-PCR showed that Magnolol suppressed epithelial-mesenchymal-transition by increasing the expression level of E-cadherin and decreasing those of $\mathrm{N}$-cadherin and vimentin. Magnolol suppressed the TGF- $\beta / S$ mad pathway by negatively regulating phosphorylation of Smad2/3. Moreover, TGF- $\beta 1$ impaired the antitumor effects of Magnolol in vivo. These results demonstrated that Magnolol can inhibit proliferation, migration and invasion in vivo and in vitro by suppressing the TGF- $\beta$ signal pathway and EMT. Magnolol could be a hopeful therapeutic drug for pancreatic malignancy.

Keywords: magnolol, pancreatic cancer, TGF- $\beta$, epithelial-mesenchymal-transition, Smad

\section{INTRODUCTION}

Pancreatic cancer is a common malignancy and ranks as the 7th leading cancer-associated mortality in developed nations (1). Although great improvement has been reached in surgery, chemotherapeutics and radiotherapeutics, the prognosis is still poor due to the local invasion and metastasis upon diagnosis $(2,3)$. Furthermore, no definite cure strategy can be provided to treat the patients with advanced stages or metastasis (2). Therefore, it is urgent to develop new and effective drugs to treat pancreatic cancer.

Epithelial-mesenchymal-transition (EMT) is an important segment in cancer invasion, metastasis, proliferation and maintenance of stem cell characteristics (4). When EMT occurs, 
tumor cells lose epithelial integrity and cell polarity and gain an invasive and motile phenotype (5). Accumulated evidence demonstrates that transforming growth factor- $\beta$ (TGF $\beta$ ) signaling is a prime inducer of EMT in the tumor microenvironment (6). The increased pretreatment of soluble TGF- $\beta 1$ in serum of unresectable pancreatic cancer patients indicates a poor prognosis for chemotherapy (7).

Magnolol (MAG, $\mathrm{C}_{18} \mathrm{H}_{18} \mathrm{O}_{2}$, CAS Number 528-43-8, PubChem CID 72300) is a natural hydroxylated biphenyl extracted from the root and stem bark of Magnolia officinalis, Magnolia obovata and Magnolia grandiflora (8). Magnolol is reported to have a good safety profile and anti-tumor effects in accumulated studies. Magnolol mediates cell death via PI3K/ Akt-mediated epigenetic modifications and benefits the management of melanoma (9). Magnolol inhibits the growth and invasion of cholangiocarcinoma cells via the NF-kappaB pathway (10). However, whether Magnolol inhibits the EMT and invasion and migration in vivo, how it restrains growth of the tumor in vitro remains unclear.

In the present study, a pancreatic orthotopic xenograft in a model nude mouse was applied to access the effect of Magnolol on the tumor growth, and pancreatic cancer cell lines (Panc-1 and AsPC-1) were employed to evaluate the impacts of Magnolol on cell biological behavior. TGF- $\beta 1$-induced EMT was also investigated as a potential mechanism of action of Magnolol.

\section{MATERIALS AND METHODS}

\section{Chemicals}

Magnolol $\left(\mathrm{C}_{18} \mathrm{H}_{18} \mathrm{O}_{2}\right.$, 5'-Diallyl-2,2'-dihydroxybiphenyl, molecular weight: 266.33 , purity $\geq 98 \%$ ) was purchased from Aladdin Company (Cat. M111378, Aladdin, Shanghai, China). Dimethyl sulfoxide (DMSO, Aladdin, Shanghai, China) was used as solvent. A stock solution of magnolol (125 mM, 332.9 $\mathrm{mg}$ magnolol in $10 \mathrm{ml} \mathrm{DMSO}$ ) was stored at $-15^{\circ} \mathrm{C}$. Magnolol stock was diluted in PBS $(1.5 \mathrm{mM}$ for magnolol, $1.2 \% \mathrm{v} / \mathrm{v}$ for DMSO) and PBS-DMSO (1.2\%) was served as a negative control. Recombinant Human Transforming Growth Factor $\beta 1$ (TGF- $\beta 1$ ) was obtained from R\&D systems (Cat. 240-B002, Shanghai, China) and dissolved with sterile $\mathrm{dd}_{2} \mathrm{O}$ when need.

\section{Cell Culture}

The human pancreatic cancer cell lines PANC-1 and AsPC-1 were obtained from American Type Culture Collection (ATCC, Manassas, VA, USA) and were cultured with DMEM medium with $10 \%$ fetal bovine serum (Gibco), $100 \mathrm{U} / \mathrm{ml}$ penicillin, and $100 \mu \mathrm{g} / \mathrm{ml}$ streptomycin in incubators under $5 \% \mathrm{CO}_{2}$ at $37^{\circ} \mathrm{C}$.

\section{CCK-8 Assay}

Approximately 2,000 cells were inoculated in each well of 96-well plates and incubated with a concentration range of Magnolol. A Cell Counting Kit-8 (ab228554, abcam, shanghai China) was applied to test the cellular viability. The experiments were performed five times independently.

\section{Colony Formation Assay}

Approximately 200 cells were seeded in each well of 6-well culture plates, and the plates were incubated for $10 \mathrm{~h}$. Then, fresh media containing $0,15,30 \mu \mathrm{M}$ Magnolol were applied to replace the old media, and the samples were then cultured for 14 days. The colonies were fixed in $4 \%$ paraformaldehyde (Aladdin, Shanghai, China) and then stained with crystal violet (Aladdin, Shanghai, China). Formatted colonies were gently washed and air seasoned before photos of them were taken. The experiments were performed three times independently.

\section{Transwell Assay for Invasion}

Transwell chambers with 24 wells and $8.0-\mu \mathrm{m}$ pore membranes (Corning USA) were applied following the manufacturer's protocol. Approximately 100,000 cells were seeded in each well in the upper chamber. The upper chamber was filled with serumfree medium which was covered by thin layers of matrigel basement membrane matrix while the lower chamber was filled with complete medium. The invaded cells were fixed with $4 \%$ paraformaldehyde and stained with $0.1 \%$ crystal violet solution after being incubated for $24 \mathrm{~h}$ at $37^{\circ} \mathrm{C}$. Photos were taken using light microscopy and the invaded cells in five random fields were counted.

\section{Scratch-Wound Migration Assay}

Cells were seeded and confluently cultured in 6-well plates. The scratches were made by scraping with a sterile 1-ml pipette tip in confluently cultured cells. The medium was changed to medium containing 0,15 , or $30 \mu \mathrm{M}$ Magnolol, and the samples were incubated for $48 \mathrm{~h}$. Photos were captured using a phase-contrast microscope at 0,24 , and $48 \mathrm{~h}$. The rate of cell migration equals the ratio of the coalesced area of the scratch in $48 \mathrm{~h}$ to total the area of the scratch at $0 \mathrm{~h}$. The experiments were performed three times independently.

\section{Evaluation of Cell Morphology}

To measure changes in cell shape during EMT, we applied the published methods (11). Photos taken by an inverted phase contrast microscope during cell culture were analyzed by Image Plus software. Cell area, major axis, minor axis of random 50 cells per condition were collected and used to calculate the hybrid morphology parameters. Roundness $(4 \times$ area $\left./(\pi \times \text { major axis })^{2}\right)$, for which a high value indicates a high degree of roundness in cell shape (11). Aspect ratio (major axis/ minor axis), for which a high value indicates an elongated cell shape (11).

\section{Western Blot}

RIPA buffer (Beyotime, China) was applied to obtain total protein from cells. Same amounts of protein were isolated by SDS-PAGE and then passed on to a polyvinylidene difluoride membrane (Millipore). The membrane was incubated with primary antibodies against E-cadherin (w101482, WanleiBio, Shenyang, China), vimentin (WL01960, WanleiBio), pSMAD2/ 3 (WL02305, WanleiBio), SMAD2/3 (WL01520, WanleiBio), and $\beta$-actin (WL10372, WanleiBio) and were then incubated with HPR-conjugated secondary antibody. Chemiluminescence 
reagents (WanleiBio) were applied to visualize Protein bands. The experiments were performed three times independently.

\section{Orthotopic Xenograft Study}

Five to six-week-old BALB/C nude mice were purchased from Shanghai SLAC Animal Center (Shanghai, China). An orthotopic xenograft nude mouse model was established by Orthotopic Injection Technique as described previously (12). Briefly, luciferase-tagged AsPC- 1 cells $\left(1 \times 10^{6} / 50 \mu \mathrm{l}\right)$ were injected into the pancreas of immunocompromised mice. Pinholes were sutured with Prolene 7-0. Once orthotopic xenograft became palpable (approximately 7 days after injection), the mice were divided into two groups randomly (four mice per group). The MAG group received i.p. injection of Magnolol (50 mg/kg, once daily), and the Control group received vehicle (equal amount of DMSO) only. Tumor growth was assessed per week by bioluminescence imaging following intravenous d-luciferin injection $(150 \mathrm{mg} / \mathrm{kg})$. Final imaging was obtained at 28 days, and then mice were sacrificed. The primary orthotopic xenografts were harvested, weighed, and fixed in Bouin's solution.

\section{Immunohistochemical Staining}

Paraffin specimens of harvested xenografts were cut into $4-\mu \mathrm{m}$ thick sections and mounted on silanized slides. The immunohistochemical staining was performed as published (13). The antibodies used were anti-Ki67 (Abcam, ab15580), anti-E-cadherin (Abcam, ab231303), anti-vimentin (Abcam, ab92547) and goat anti-rabbit secondary antibody (Abcam, ab205719). Finally, 3,3-diaminobenzidine was applied to visualize the results, and photographs were taken under a microscope $(\times 100$ and $\times 400)$.

\section{Statistical Analysis}

All data are expressed as the mean \pm SD and were analyzed using Student's t test or one-way ANOVA where necessary. GraphPad Prism V6 was applied to analyze and visualize the data. All experiments were performed independently at least 3 times. A P value more than 0.05 was defined as statistically significant.

\section{RESULTS}

\section{MAG Inhibits Pancreatic Orthotopic Xenograft Growth in a Mouse Model}

We assessed the antitumor effect of MAG with a pancreatic orthotopic xenograft in a mouse model. We chose AsPC-1 for its high tumorigenicity in mice. The experimental design is shown in Figure 1A. The living imaging analysis revealed that xenograft progression with MAG treatment was significantly slower compared with that in the control group (Figures 1B, C). Additionally, the final harvested xenografts had an average weight of $0.46 \mathrm{~g}$ in MAG group compared with $0.28 \mathrm{~g}$ in the control group (Figures 1D, E). There were no differences among groups besides tumor size, while no signs of toxicity.

\section{EMT and Proliferation Is Restrained in Pancreatic Xenografts of MAG-Treated Mice}

EMT plays a critical role in cancer proliferation and maintenance of stem cell characteristics, which is reported to be significant from experimental and clinical points of view (4). We further assessed the effect of MAG on the EMT and proliferation in harvested xenografts. The proliferation and EMT was assessed using immunohistochemical staining for Ki67, E-cadherin and vimentin which are widely accepted markers (14-16). The results showed intense staining of Ki67 and vimentin in xenograft sections from the control group, whereas weak staining was detected in the MAG group (Figures 2A, B, D). Furthermore, strong staining of E-cadherin was detected in xenograft sections from the MAG group, while weak staining was detected in the control group (Figures 2A, C). These results indicate that MAG restrains EMT and proliferation in pancreatic xenografts in mice.

\section{MAG Suppressed the Viability of Pancreatic Cancer Cells}

The impact of Magnolol on the viability of PANC-1 and AsPC-1 cells was determined using an MTT assay. PANC-1 and AsPC-1 cells were incubated with a concentration range of Magnolol for 24, 48, and $72 \mathrm{~h}$. The data revealed that Magnolol suppressed the viability of these cells both time- and concentration-dependently (Figures 3A, D). Respectively, the half inhibition concentrations (IC50) on Panc- 1 cells were $140.5,117.3$, and $96.4 \mu \mathrm{M}$ for 24,48 , and $72 \mathrm{~h}$, while the IC50 values on the AsPC-1 cells were 160.0, 104.2 , and $75.4 \mu \mathrm{M}$. The IC20 and IC10 of Magnolol of Panc-1 cell were 30.7 and $14.0 \mu \mathrm{M}$ for $48 \mathrm{~h}$, while those of the AsPC-1 cells were 24.6 and 10.6 $\mu \mathrm{M}$. Panc-1 and AsPC-1 cells incubated with Magnolol at 15 and $30 \mu \mathrm{M}$ for 14 days showed compromised capability in colony formation assay (Figures 3B, C, E, F).

\section{Magnolol Suppressed Cellular Migration and Invasion In Vitro}

The scratch-wound assay showed that Magnolol treatment weakened the migration of cancer cells (Figures 4A-D). MAG-treated cells displayed epithelial-like phenotype while untreated cells showed mesenchymal-like phenotype in morphology (Figure 4E). The treatment of Magnolol $(30 \mu \mathrm{M})$ in PANC-1 and $(15 \mu \mathrm{M} \& 30 \mu \mathrm{M})$ in AsPC-1 caused significant increase in Roundness and decrease in Aspect ratio (Figures 4G, H). Magnolol treatment reduced the invasive rate of PANC-1 and AsPC-1 cells in transwell invasion assay (Figures 4F, I). These findings indicated that the inhibiting effects of Magnolol on migration and invasion may be related to EMT.

\section{Magnolol Inhibited EMT via the Suppression of TGF- $\beta 1 /$ Smad Signaling}

TGF- $\beta 1$ is the main inducer of EMT in the tumor microenvironment (17). To further explore the mechanism of action, the expression levels of EMT-associated genes and TGF- $\beta$ signaling were detected in Magnolol-treated cells. Western blot showed elevated expression of E-cadherin and reduced expression of Vimentin in Magnololtreated cells at the protein level (Figures 5A-C). Considering that 

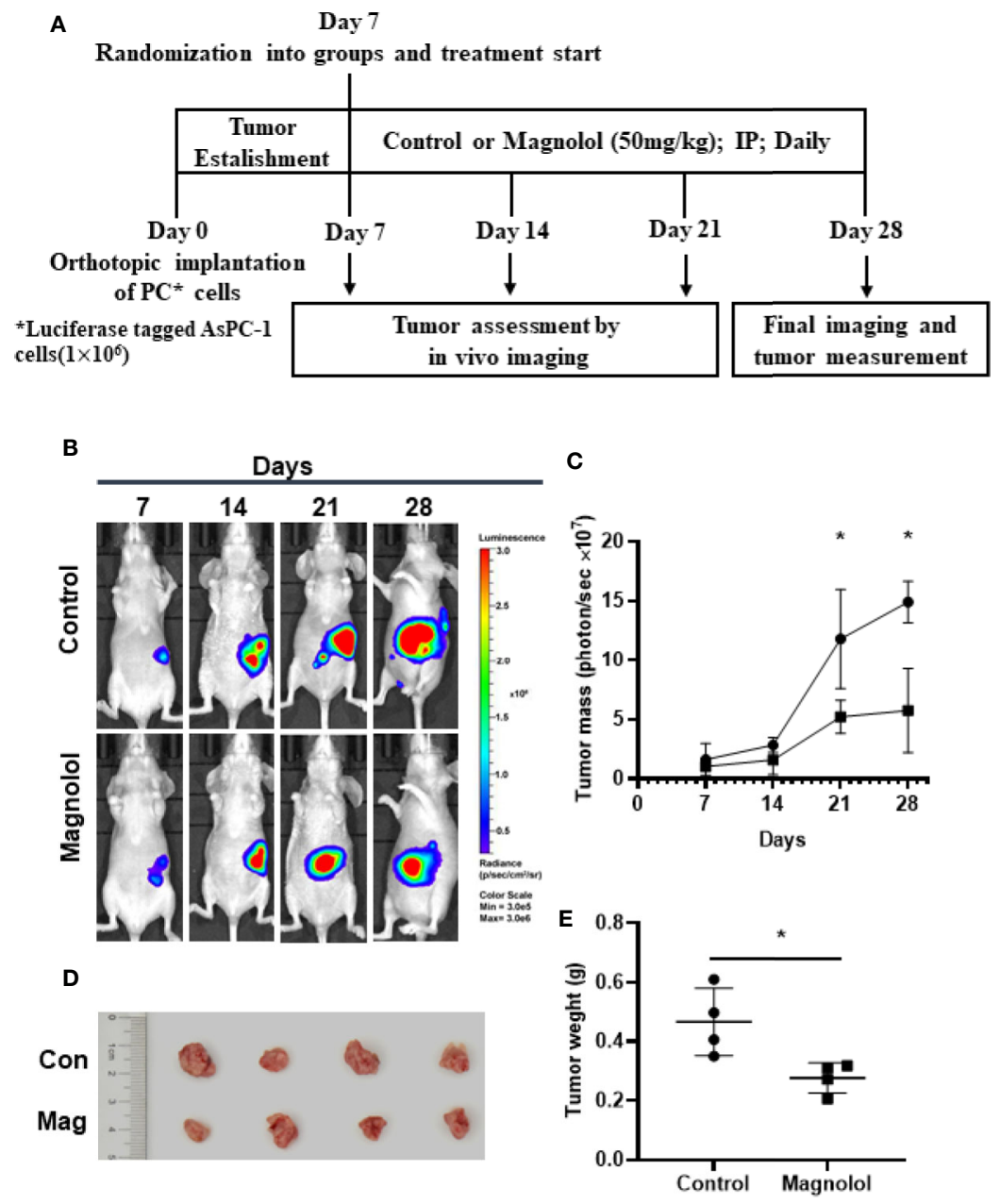

FIGURE 1 | Magnolol (MAG) inhibits pancreatic orthotopic xenograft growth in a mouse model. (A) Schematic representation of in vivo treatment strategy. (B) Images by the living imaging analysis of mice from both the groups at different time points. (C) Tumor growth curve (total photons per second) showing tumor growth at different time points. (D) The image and (E) weight of the tumors harvested at the end point. ${ }^{\star} P<0.05$.

the phosphorylation of Smads is a critical event in the TGF- $\beta$ signaling, we determined the phosphorylation level of Smad $2 / 3$ in Magnolol-treated cells using western blot. The results showed that Magnolol reduced the phosphorylation of Smad2/3 in PANC-1 and AsPC-1 cells (Figures 5A-C).

\section{Magnolol Abolished TGF- $\beta 1$-Induced Migration and Invasion In Vitro}

Because Magnolol restrained the migration, invasion and altered the cell morphology, we further explored whether these effects of Magnolol relied on TGF- $\beta 1$-induced EMT. Cancer cells were incubated with $10 \mathrm{ng} / \mathrm{ml}$ TGF- $\beta 1$ cytokine alone or with $15 \mu \mathrm{M}$ of Magnolol for $48 \mathrm{~h}$. TGF- $\beta 1$ significantly promoted the migration and invasion and Magnolol markedly impaired these effects of TGF- $\beta 1$ in the PANC- 1 and AsPC- 1 cells (Figure 6). The morphological characteristics these cells were more mesenchymal after being induced by $10 \mathrm{ng} / \mathrm{ml}$ TGF- $\beta 1$ alone than when incubated with $10 \mathrm{ng} / \mathrm{ml}$ TGF- $\beta 1$ cytokine and $15 \mu \mathrm{M}$
Magnolol (Figure 7A). The Roundness was significantly decreased while the Aspect ratio was significantly increased after being induced by $10 \mathrm{ng} / \mathrm{ml}$ TGF- $\beta 1$ (Figure 7B). These effects were abolished by $15 \mu \mathrm{M}$ Magnolol treatment (Figure 7B). Western blot showed that incubation of cancer cells with Magnolol and TGF- $\beta 1$ simultaneously markedly decreased the levels of E-cadherin and phosphorylation of Smad2/3 compared with incubation with TGF- $\beta 1$ alone (Figures 7C-F). These results demonstrated that Magnolol suppressed the aggressive behavior of these cells by inhibiting TGF- $\beta 1$-induced EMT.

\section{DISCUSSION}

Current study was aimed to explore the therapeutic effect of Magnolol on pancreatic cancer in vivo and in vitro. Magnolol inhibited pancreatic tumor development in mouse model and suppressed the aggressive behavior of pancreatic cancer cells in a 

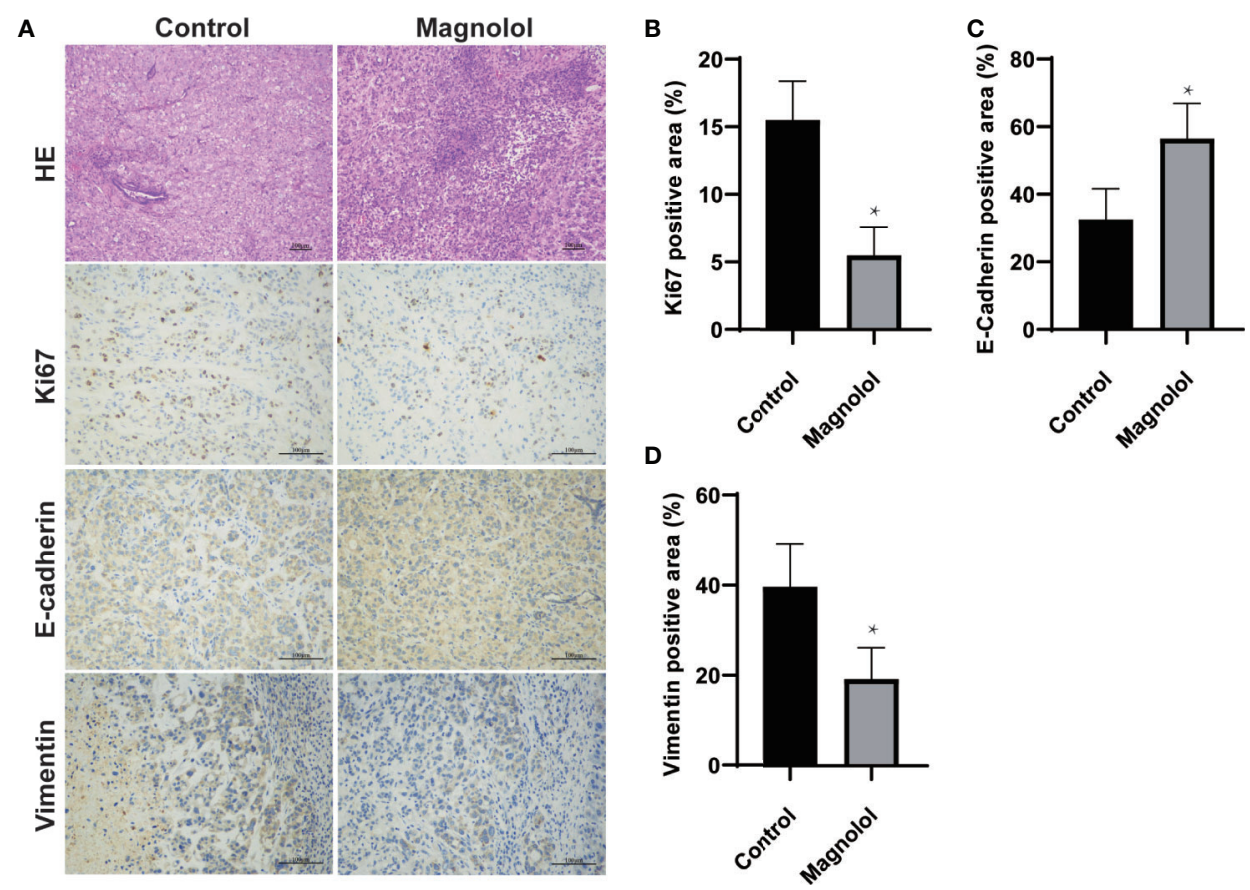

FIGURE 2 | Epithelial-mesenchymal-transition (EMT) and proliferation is restrained in pancreatic xenografts of Magnolol (MAG)-treated mice. (A) HE staining was applied to confirm the xenografts formation while Immunohistochemical staining was used to access the expression of Ki67, E-cadherin and vimentin. (B-D) The expression of Ki67, E-cadherin and vimentin were analyzed. ${ }^{\star} P<0.05$.

A

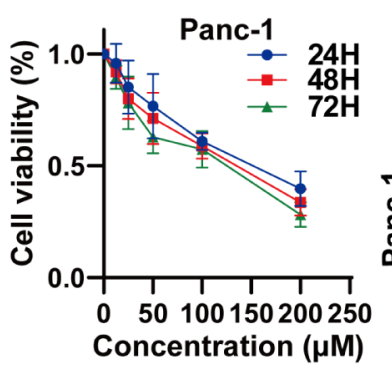

D

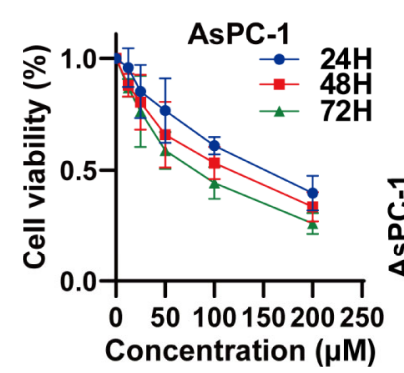

B

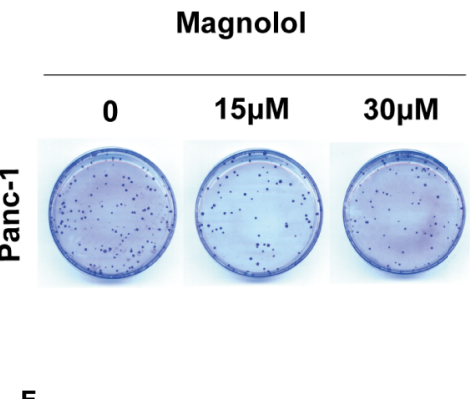

E

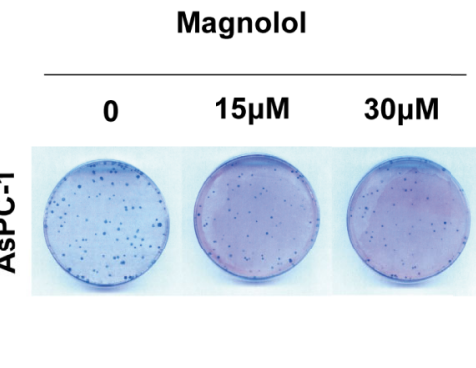

C

Panc-1

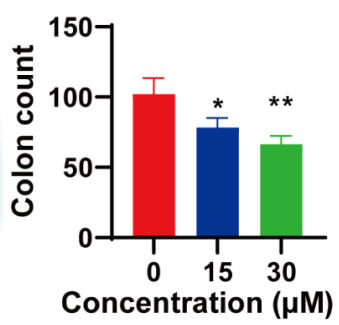

$\mathbf{F}$

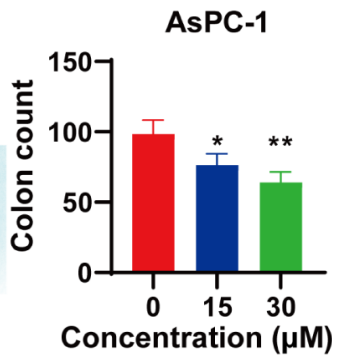

FIGURE 3 | Magnolol (MAG) suppressed the viability of pancreatic cancer cells (A, D) MAG suppressed the viability of Panc-1 and AsPC-1 in time- and concentration-dependent manner. (B, C, E, F) MAG $(15$ or $30 \mu \mathrm{M})$ reduced colony formation of Panc-1 and AsPC-1. ${ }^{*} P<0.05 ;{ }^{* *} P<0.01$. 


\section{A}
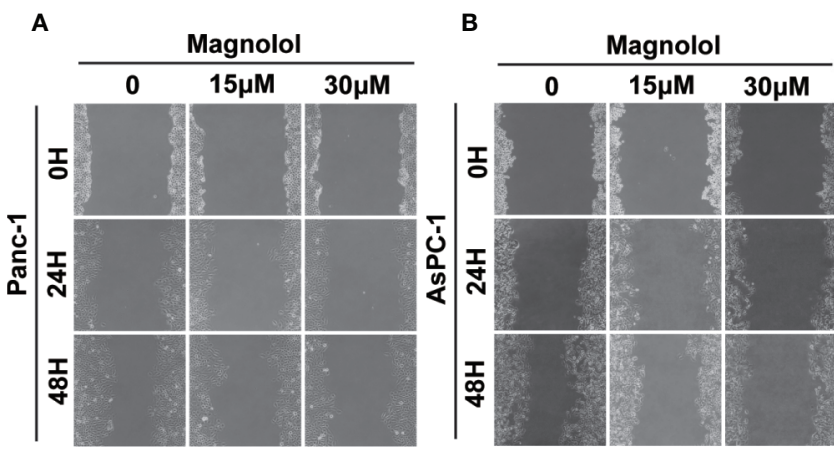

C

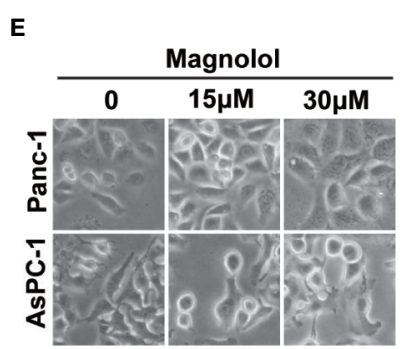

G

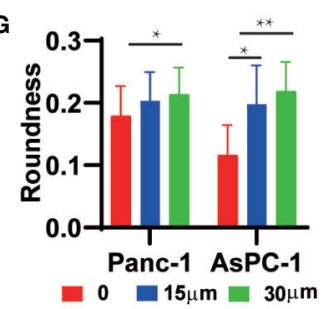

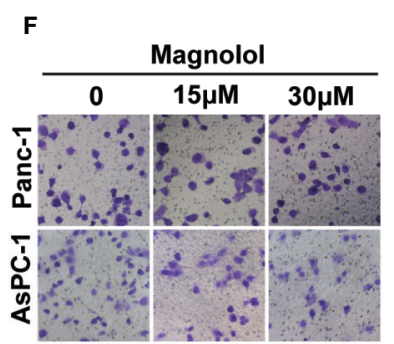

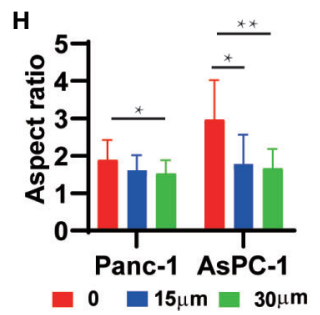

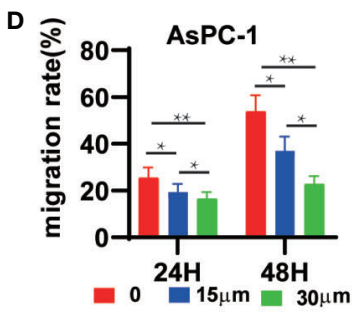
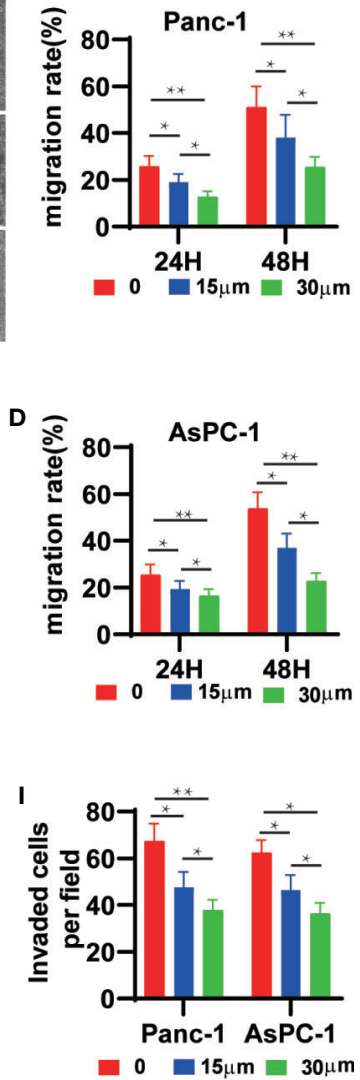

FIGURE 4 | Magnolol Suppressed Cellular Migration and Invasion in vitro (A, B) The wound-healing of cells treated with Magnolol(0, 15 , $30 \mu \mathrm{M})$ was shown at 0, 24 and $48 \mathrm{~h}$ after scratching. (C, D) The results of the scratch-wound assay were analyzed. (E) Morphological changes in Panc-1 and AsPC-1 cells after culture with Magnolol(0, 15, $30 \mu \mathrm{M})$. (G, H) Evaluation of cell morphology for Panc-1 and AsPC-1 cells after culture with Magnolol(0, 15, $30 \mu \mathrm{M})$. (F, I) Transwell invasion assay showed Magnolol treatment reduced the invasive rate of PANC-1 and AsPC-1 cells. ${ }^{*} \mathrm{P}<0.05 ;{ }^{* *} \mathrm{P}<0.01$.

time- and concentration-dependent manner. As far as we know, the current study is the first to demonstrate these effects of Magnolol on pancreatic cancer. These data suggested that Magnolol inhibited TGF- $\beta 1$-induced EMT via negatively regulating the phosphorylation of Smad2/3. Thus, we recommend Magnolol as a potential therapeutic for the treatment of pancreatic cancer. Further study will be needed to confirm molecular targets and loci.

Metastasis is a hallmark of tumors that contributes to cancerassociated death (18). The majority of patients are found with metastasis when diagnosed with pancreatic cancer (19). Tumor metastasis refers to a multi-step process of tumor cell migration from the primary site to distant sites (4). Epithelial-tomesenchymal transition (EMT) features the evanishment of adhesion between cells accompanied by elevated levels of epithelial markers and reduced levels of mesenchymal markers (4). A series of pathways including Wnt, $\beta$-catenin, Notch, and tyrosine kinases is related to the induction of EMT. Among them, TGF- $\beta 1$ is known as not only an inflammatory cytokine but also a pivotal inducer and sustainer of EMT (20). EMT is closely involved in not only invasion and metastasis but also proliferation and chemotherapy resistance. TGF- $\beta$-induced EMT plays an important role in PDAC progression, especially in metastases (20). Once bonded to its receptor on the cytomembrane, TGF- $\beta$ induces phosphorylation of its downstream factors, including but not limited to SMAD proteins. The phosphorylated SMAD2/3 and SMAD4 subsequently form a regulatory complex that promotes the transcription of target genes (21).

Magnolol is a natural neolignan from magnolia plants and is reported to present antitumor, anti-oxidative, antiinflammatory, and antibacterial effects (8). The antitumor properties of magnolol were reported to be related but not limited to the PI3K-AKT, p53, NF- $\kappa \mathrm{B}$, and p53-mediated mitochondrial pathways $(9,10,22,23)$. Unlike well-reported anti-proliferative effects, the anti-metastasis effects of magnolol are not as well studied. Magnolol has been reported to inhibit the ERK-modulated metastatic potential of hepatocellular carcinoma cells (24) and the HER2-modulated metastatic potential of ovarian cancer cells (25). Moreover, magnolol suppresses 

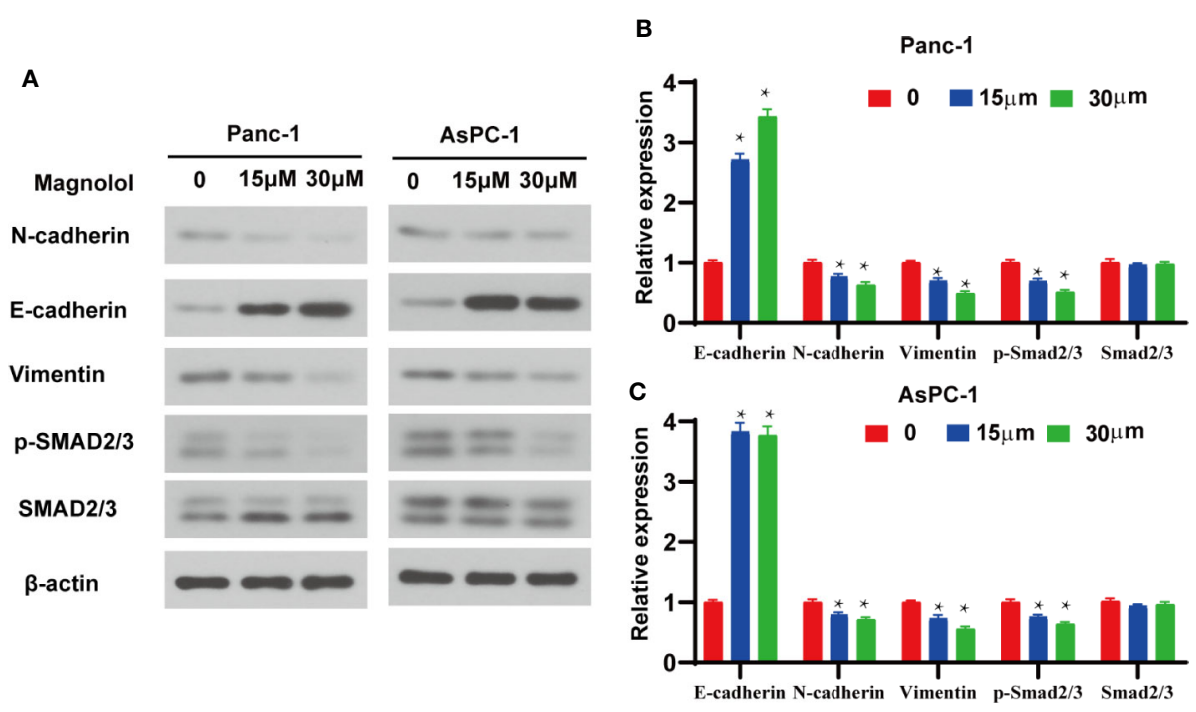

FIGURE 5 | Magnolol inhibited epithelial-mesenchymal-transition (EMT) via the suppression of transforming growth factor- $\beta 1$ (TGF- $\beta 1$ )/Smad Signalling. (A) Western blot analysis of protein levels of E-cadherin, $\mathrm{N}$-cadherin, Vimentin, $\mathrm{p}$-Smad2/3, Smad2/3 in Panc-1 and AsPC-1 cells treated with Magnolol(0, 15 , 30 $\mu$ M) for 48 h. $(\mathbf{B}, \mathbf{C})$ Histograms show the change of relative protein expression of E-cadherin, N-cadherin, Vimentin, p-Smad2/3, Smad2/3 in Panc-1 and AsPC-1 cells treated with Magnolol(0, 15, $30 \mu \mathrm{M})$ for $48 \mathrm{~h} .{ }^{*} \mathrm{P}<0.05$.
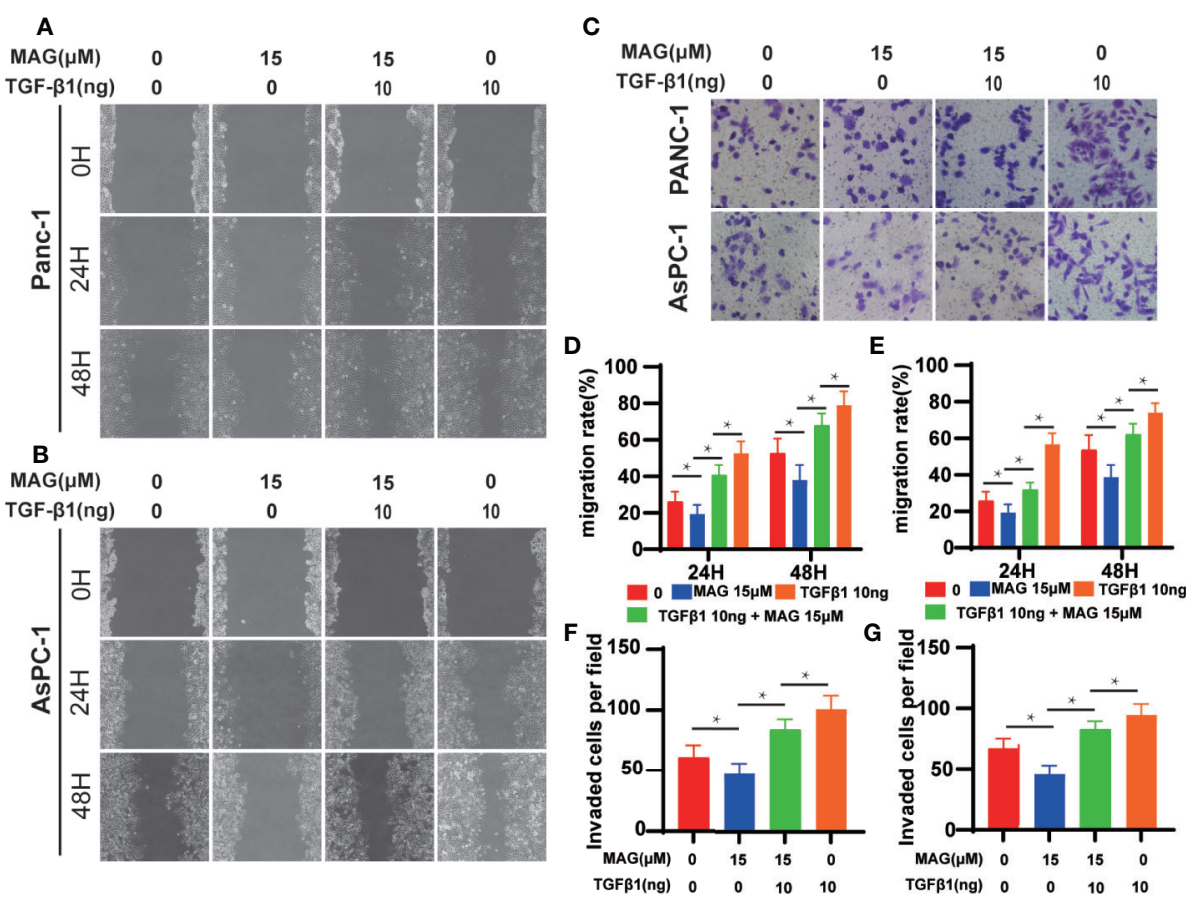

FIGURE 6 | Magnolol abolished transforming growth factor- $\beta 1$ (TGF- $\beta 1$ )-Induced Migration and Invasion in vitro. (A, B) The wound-healing of cells treated with with Magnolol or TGF- $\beta 1$ was shown at 0,24 , and 48 h after scratching. (D, E) The migration rate of the scratch-wound assay was analyzed. (E) Morphological changes in Panc- 1 and AsPC-1 cells after culture with Magnolol or TGF- $\beta 1$. (C, F, G) Transwell invasion assay showed Magnolol treatment abolished TGF- $\beta 1$ induced invasion of PANC-1 and AsPC-1 cells. * $\mathrm{P}<0.05$. 

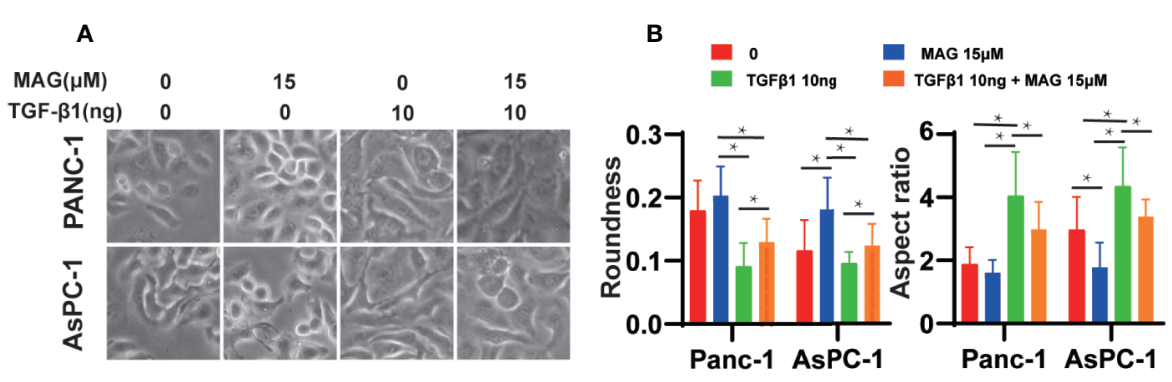

C

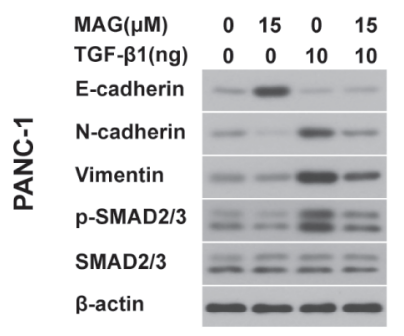

E

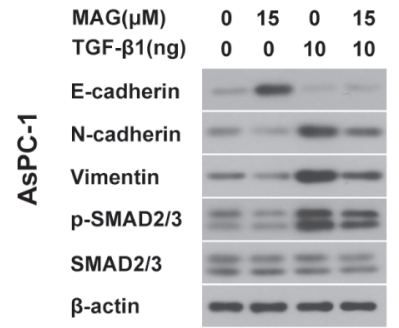

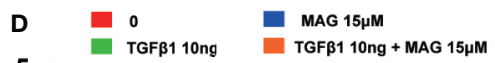
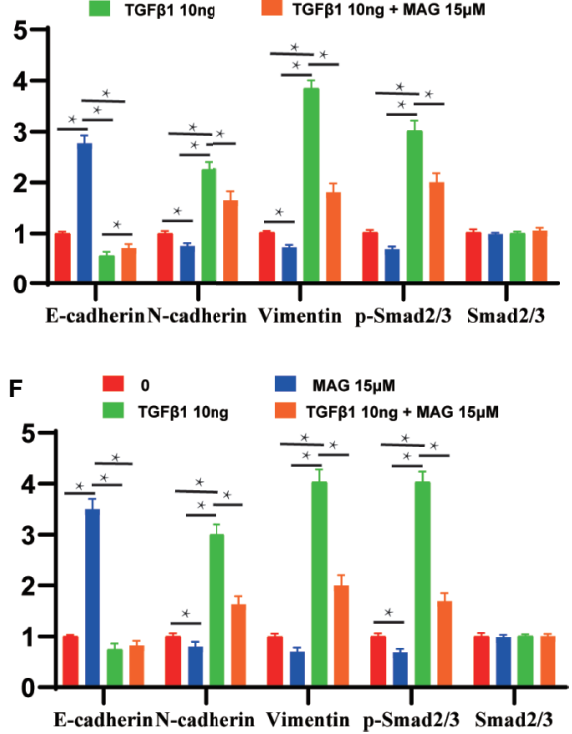

FIGURE 7 | Magnolol abolished transforming growth factor- $\beta 1$ (TGF- $\beta 1$ )-Induced epithelial-mesenchymal-transition (EMT) amd phosphorylation of Smad2/3 in vitro. (A) Morphological changes in Panc-1 and AsPC-1 cells after culture with Magnolol or TGF- $\beta 1$. (B) Evaluation of cell morphology for Panc-1and AsPC-1 treated with Magnolol or TGF- $\beta 1$. (C, E) Western blot results of E-cadherin, N-cadherin, Vimentin, p-Smad2/3, Smad2/3 in Panc-1or AsPC- 1 treated with Magnolol or TGF- $\beta 1$. (D, F) Histograms show the changes of relative expression of E-cadherin, N-cadherin, Vimentin, p-Smad2/3, Smad2/3 in Panc-1or AsPC-1 treated with Magnolol or TGF- $\beta 1 .{ }^{*} \mathrm{P}<0.05$.

metastasis via matrix metalloproteinase-2/-9 activities in prostate carcinoma cells (26). However, the anti-metastatic function of magnolol remains unclear, especially in pancreatic cancer. In default of known research on this issue, we conducted this research.

The TGF- $\beta /$ Smad signaling pathway is related to the occurrence of neurological disorders, fibrosis and malignancies (6). The TGF- $\beta$ pathway presents a growth-repressive effect in the early stage of tumorigenesis in the pancreas by facilitating cell cycle arrest and apoptosis. Conversely, TGF- $\beta$ signaling accelerates tumor progression in advanced-stage pancreatic cancer by inducing EMT, metastasis, invasion, migration, and immune evasion (27). In fact, the contribution of TGF $\beta$ to metastases has been well proven in pancreatic cancer $(28,29)$. Zhang et al. showed that Magnolol reduces bleomycin-induced rodent lung fibrosis by reducing TGF- $\beta 1$ expression (30). Chei et al. reported that Magnolol does not influence apoptosis, but restrains cell migration, invasion and EMT in human colorectal tumor cells via suppressing the TGF- $\beta$ pathway (31).
In the current study, we explored the effects of magnolol on pancreatic cancer in vivo and in vitro. First, the anti-tumor effect of magnolol was verified using orthotopic xenograft, and the changes in the expression of EMT markers were detected using immunohistochemical staining. Second, we studied the role of magnolol in impairing TGF- $\beta 1$-mediated migration, invasion, proliferation and EMT in pancreatic cancer cells. Cancer cells were cultured with different concentrations of magnolol for 24, 48 , and $72 \mathrm{~h}$ to explore the concentration-effect relationship. We chose concentrations of magnolol that suppress migration and invasion without inducing apparent cell proliferation arrest to perform our experiments. As published, $10 \mathrm{ng} / \mathrm{ml}$ of TGF- $\beta 1$ induced EMT and facilitated pancreatic cancer cell migration and invasion $(32,33)$. We confirmed the EMT-inductive effects of that concentration in pancreatic cancer cells and then used it in our experiments. Our study verified that EMT played an important role in the aggressive actions of pancreatic cancer, while magnolol suppressed the migration, invasion, and proliferation of pancreatic cancer via EMT in vivo and in vitro. 
There are also some limitations in our research. First, we studied the anti-tumor effects of magnolol on EMT by the Smaddependent TGF- $\beta$ pathway; however, the Smad-independent TGF- $\beta$ pathway or other signaling pathways related to EMT and cancer were not detected. Second, while we found that magnolol suppressed phosphorylation of SMAD2/3, we did not verify the responsible molecular site of action. Third, we observed the tumor-suppressing effect of magnolol in vitro, and we focused on cancer cells to explore the antitumor effects of magnolol, but the effect of magnolol on the tumor environment also needs investigation. It will be necessary to perform in-depth research on the effects and mechanism of magnolol to determine whether magnolol could be used in the treatment of pancreatic cancer.

\section{DATA AVAILABILITY STATEMENT}

The raw data supporting the conclusions of this article will be made available by the authors, without undue reservation.

\section{REFERENCES}

1. Siegel RL, Miller KD, Jemal A. Cancer statistics, 2019. CA Cancer J Clin (2019) 69:7-34. doi: 10.3322/caac.21551

2. Peixoto RD, Speers C, McGahan CE, Renouf DJ, Schaeffer DF, Kennecke HF. Prognostic factors and sites of metastasis in unresectable locally advanced pancreatic cancer. Cancer Med (2015) 4:1171-7. doi: 10.1002/ cam 4.459

3. Von Hoff DD, Ervin T, Arena FP, Chiorean EG, Infante J, Moore M, et al. Increased survival in pancreatic cancer with nab-paclitaxel plus gemcitabine. N Engl J Med (2013) 369:1691-703. doi: 10.1056/NEJMoa1304369

4. Tanabe S, Quader S, Cabral H, Ono R. Interplay of EMT and CSC in Cancer and the Potential Therapeutic Strategies. Front Pharmacol (2020) 11:904. doi: 10.3389/fphar.2020.00904

5. Faheem MM, Seligson ND, Ahmad SM, Rasool RU, Gandhi SG, Bhagat M, et al. Convergence of therapy-induced senescence (TIS) and EMT in multistep carcinogenesis: current opinions and emerging perspectives. Cell Death Discov (2020) 6:51. doi: 10.1038/s41420-020-0286-Z

6. Batlle E, Massague J. Transforming Growth Factor-beta Signaling in Immunity and Cancer. Immunity (2019) 50:924-40. doi: 10.1016/ j.immuni.2019.03.024

7. Park H, Bang JH, Nam AR, Park JE, Jin MH, Bang YJ, et al. The prognostic role of soluble TGF-beta and its dynamics in unresectable pancreatic cancer treated with chemotherapy. Cancer Med (2020) 9:43-51. doi: 10.1002/ cam4.2677

8. Ranaware AM, Banik K, Deshpande V, Padmavathi G, Roy NK, Sethi G, et al. Magnolol: A Neolignan from the Magnolia Family for the Prevention and Treatment of Cancer. Int J Mol Sci (2018) 19:2362. doi: 10.3390/ijms19082362

9. Emran AA, Chinna Chowdary BR, Ahmed F, Hammerlindl H, Huefner A, Haass NK, et al. Magnolol induces cell death through PI3K/Akt-mediated epigenetic modifications boosting treatment of BRAF- and NRAS-mutant melanoma. Cancer Med (2019) 8:1186-96. doi: 10.1002/cam4.1978

10. Zhang FH, Ren HY, Shen JX, Zhang XY, Ye HM, Shen DY. Magnolol suppresses the proliferation and invasion of cholangiocarcinoma cells via inhibiting the NF-kappaB signaling pathway. Biomed Pharmacother Biomed Pharmacother (2017) 94:474-80. doi: 10.1016/j.biopha.2017.07.085

11. Uynuk-Ool T, Rothdiener M, Walters B, Hegemann M, Palm J, Nguyen P, et al. The geometrical shape of mesenchymal stromal cells measured by quantitative shape descriptors is determined by the stiffness of the biomaterial and by cyclic tensile forces. J Tissue Eng Regener Med (2017) 11:3508-22. doi: $10.1002 /$ term. 2263

\section{ETHICS STATEMENT}

The animal study was reviewed and approved by animal ethics committee of Xi'an Jiaotong University.

\section{AUTHOR CONTRIBUTIONS}

All authors listed have made a substantial, direct, and intellectual contribution to the work and approved it for publication.

\section{FUNDING}

The project was supported by National Natural Science Foundation of China, NSFC (No:82003998, 81602401); Natural Science Foundation of Shaanxi Province (No: 2019JQ-969); and the Xi'an Jiaotong University Education Foundation, XJTUEF (No: $\mathrm{xjj2018141).}$

12. Hotz HG, Reber HA, Hotz B, Yu T, Foitzik T, Buhr HJ, et al. An orthotopic nude mouse model for evaluating pathophysiology and therapy of pancreatic cancer. Pancreas (2003) 26:e89-98. doi: 10.1097/00006676200305000-00020

13. Shan T, Chen S, Chen X, Lin W, Li W, Ma J, et al. Association of family history of tumors with clinicopathological characteristics and prognosis of colorectal cancer. Eur J Cancer Prev (2019) 28:258-67. doi: 10.1097/CEJ.0000000000000482

14. Menon SS, Guruvayoorappan C, Sakthivel KM, Rasmi RR. Ki-67 protein as a tumour proliferation marker. Clin Chim Acta (2019) 491:39-45. doi: 10.1016/ j.cca.2019.01.011

15. Sommariva M, Gagliano N. E-Cadherin in Pancreatic Ductal Adenocarcinoma: A Multifaceted Actor during EMT. Cells (2020) 9:1040. doi: $10.3390 /$ cells 9041040

16. Strouhalova K, Prechova M, Gandalovicova A, Brabek J, Gregor M, Rosel D. Vimentin Intermediate Filaments as Potential Target for Cancer Treatment. Cancers (Basel) (2020) 12:181. doi: 10.3390/cancers 12010184

17. Dudas J, Ladanyi A, Ingruber J, Steinbichler TB, Riechelmann H. Epithelial to Mesenchymal Transition: A Mechanism that Fuels Cancer Radio/ Chemoresistance. Cells (2020) 9:428. doi: 10.3390/cells9020428

18. Ksiazkiewicz M, Markiewicz A, Zaczek AJ. Epithelial-mesenchymal transition: a hallmark in metastasis formation linking circulating tumor cells and cancer stem cells. Pathobiology (2012) 79:195-208. doi: 10.1159/ 000337106

19. Zhao C, Gao F, Li Q, Liu Q, Lin X. The Distributional Characteristic and Growing Trend of Pancreatic Cancer in China. Pancreas (2019) 48:309-14. doi: 10.1097/MPA.0000000000001222

20. Dardare J, Witz A, Merlin JL, Gilson P, Harle A. SMAD4 and the TGFbeta Pathway in Patients with Pancreatic Ductal Adenocarcinoma. Int J Mol Sci (2020) 21:3534. doi: 10.3390/ijms21103534

21. Chen Y, Di C, Zhang X, Wang J, Wang F, Yan JF, et al. Transforming growth factor beta signaling pathway: A promising therapeutic target for cancer. J Cell Physiol (2020) 235:1903-14. doi: 10.1002/jcp.29108

22. Li M, Zhang F, Wang X, Wu X, Zhang B, Zhang N, et al. Magnolol inhibits growth of gallbladder cancer cells through the p53 pathway. Cancer Sci (2015) 106:1341-50. doi: $10.1111 /$ cas. 12762

23. Zhou S, Wen H, Li H. Magnolol induces apoptosis in osteosarcoma cells via G0/G1 phase arrest and p53-mediated mitochondrial pathway. J Cell Biochem (2019) 120:17067-79. doi: 10.1002/jcb.28968

24. Kuan LY, Chen WL, Chen JH, Hsu FT, Liu TT, Chen WT, et al. Magnolol Induces Apoptosis and Inhibits ERK-modulated Metastatic Potential in 
Hepatocellular Carcinoma Cells. Vivo (Athens Greece) (2018) 32:1361-8. doi: 10.21873/invivo.11387

25. Chuang TC, Hsu SC, Cheng YT, Shao WS, Wu K, Fang GS, et al. Magnolol down-regulates HER2 gene expression, leading to inhibition of HER2mediated metastatic potential in ovarian cancer cells. Cancer Lett (2011) 311:11-9. doi: 10.1016/j.canlet.2011.06.007

26. Hwang ES, Park KK. Magnolol suppresses metastasis via inhibition of invasion, migration, and matrix metalloproteinase-2/-9 activities in PC-3 human prostate carcinoma cells. Biosci Biotechnol Biochem (2010) 74:961-7. doi: 10.1271/bbb.90785

27. Zhang Y, Li JH, Yuan QG, Cao G, Yang WB. Upregulation of LASP2 inhibits pancreatic cancer cell migration and invasion through suppressing TGF-beta-induced EMT. J Cell Biochem (2019) 120:136517. doi: $10.1002 / \mathrm{jcb} .28638$

28. Hong E, Park S, Ooshima A, Hong CP, Park J, Heo JS, et al. Inhibition of TGF-beta signalling in combination with nal-IRI plus 5-Fluorouracil/ Leucovorin suppresses invasion and prolongs survival in pancreatic tumour mouse models. Sci Rep (2020) 10:2935. doi: 10.1038/s41598020-59893-5

29. Ottaviani S, Stebbing J, Frampton AE, Zagorac S, Krell J, de Giorgio A, et al. TGF-beta induces miR-100 and miR-125b but blocks let-7a through LIN28B controlling PDAC progression. Nat Commun (2018) 9:1845. doi: 10.1038/ s41467-018-03962-x

30. Zhang H, Ju B, Zhang X, Zhu Y, Nie Y, Xu Y, et al. Magnolol Attenuates Concanavalin A-induced Hepatic Fibrosis, Inhibits CD4(+) T Helper 17 (Th17) Cell Differentiation and Suppresses Hepatic Stellate Cell Activation:
Blockade of Smad3/Smad4 Signalling. Basic Clin Pharmacol Toxicol (2017) 120:560-70. doi: 10.1111/bcpt.12749

31. Chei S, Oh HJ, Song JH, Seo YJ, Lee K, Lee BY. Magnolol Suppresses TGFbeta-Induced Epithelial-to-Mesenchymal Transition in Human Colorectal Cancer Cells. Front Oncol (2019) 9:752. doi: 10.3389/fonc.2019.00752

32. Ellenrieder V, Hendler SF, Boeck W, Seufferlein T, Menke A, Ruhland C, et al. Transforming growth factor betal treatment leads to an epithelialmesenchymal transdifferentiation of pancreatic cancer cells requiring extracellular signal-regulated kinase 2 activation. Cancer Res (2001) 61:4222-8.

33. Wang H, Wu J, Zhang Y, Xue X, Tang D, Yuan Z, et al. Transforming growth factor $\beta$-induced epithelial-mesenchymal transition increases cancer stem-like cells in the PANC-1 cell line. Oncol Lett (2012) 3:229-33. doi: 10.3892/ ol.2011.448

Conflict of Interest: The authors declare that the research was conducted in the absence of any commercial or financial relationships that could be construed as a potential conflict of interest.

Copyright $\odot 2020$ Chen, Shen, Zhao, Wang, Shan, Li, Xu, Chen, Liu and Cao. This is an open-access article distributed under the terms of the Creative Commons Attribution License (CC BY). The use, distribution or reproduction in other forums is permitted, provided the original author $(s)$ and the copyright owner(s) are credited and that the original publication in this journal is cited, in accordance with accepted academic practice. No use, distribution or reproduction is permitted which does not comply with these terms. 